\title{
Prevalence of Musculoskeletal Pain among Dental Students
}

\author{
Nurul Syamimi Binti Mohd Azlan Sunil'1, Dhanraj Ganapathy ${ }^{2}$ and Revathi Duraisamy ${ }^{3}$ \\ ${ }^{1}$ Saveetha Dental College and Hospitals, Saveetha Institute of Medical \\ and Technical Sciences, Saveetha University, Chennai, India \\ ${ }^{2}$ Professor and Head, Department of Prosthodontics, Saveetha Dental College and Hospitals, \\ Saveetha Institute of Medical and Technical Sciences, Saveetha University, Chennai, India \\ ${ }^{3}$ Senior Lecturer, Saveetha Dental College and Hospitals, Saveetha Institute of Medical and \\ Technical Sciences, Saveetha University, Chennai, India
}

\section{ABSTRACT}

One of the professions with high incidence of musculoskeletal disorder in the course of their professional career are dentists. Musculoskeletal disorders among dental professionals start as early as during the course of clinical training period at dental school. The aim of this study was to evaluate the prevalence of musculoskeletal pain among dental students. The survey included the distribution of questionnaires to dental students from a private dental hospital during a one-week period. The criterion for selection was third to fifth year undergraduate dental student. The questionnaire included information on age and gender, and other variables such as year of study, presence of musculoskeletal pain, location of pain, characteristic of pain such as pain duration and intensity and frequency of weekly exercise. Data was analysed using SPSS Software and a chi-square test was done to find out association between variables. A total of 114 students participated in the study. 71.1\% experienced musculoskeletal pain and this was more common in females $(80.5 \%)$. The percentage of participants reporting pain appeared to increase with the number of years in dental school . The order of most prevalent pain locations was the neck/shoulder (44.4\%), lower back (28.4\%), thumb/hand (14.8\%), upper back (9.9\%) and wrist (2.5\%). The order of most prevalent pain locations was the neck/shoulder (44.4\%), lower back (28.4\%), thumb/hand (14.8\%), upper back (9.9\%) and wrist (2.5\%). Regular exercise was associated with alleviation of some pain. Within the limits of this study, it was observed that there was a high prevalence of musculoskeletal pain among dental students and prevalence was higher among females and dental interns. The most common area affected by pain was the neck/shoulder region.

KEY WORDS: BODY PAIN, DENTAL STUDENTS, EXERCISE, MUSCULOSKELETAL PAIN, NECK PAIN.

\section{ARTICLE INFORMATION}

*Corresponding Author: dhanraj@saveetha.com

Received 24th July 2020 Accepted after revision 23rd Sep 2020

Print ISSN: 0974-6455 Online ISSN: 2321-4007 CODEN: BBRCBA

Thomson Reuters ISI Web of Science Clarivate Analytics USA and Crossref Indexed Journal

$$
\begin{aligned}
& \text { Clarivate } \\
& \text { Analytics }
\end{aligned}
$$

NAAS Journal Score 2020 (4.31) SJIF: 2020 (7.728)

A Society of Science and Nature Publication,

Bhopal India 2020. All rights reserved.

Online Contents Available at: http//www.bbrc.in/

Doi: http://dx.doi.org/10.21786/bbrc/13.8/164 


\section{INTRODUCTION}

Musculoskeletal disorders are characterized by the presence of discomfort, persistent pain or disability of the joints, tendons, and muscles, aggravated or caused by repeated movements, static work postures or forced body postures. One of the professions with high incidence of musculoskeletal disorder in the course of their professional career are dentists (Pollack, 1996; Leggat, Kedjarune and Smith, 2007; Hayes, Cockrell and Smith, 2009). Musculoskeletal disorder has also been documented for dental hygienists and dental assistants (Murtomaa, 1982; Stockstill et al., 1993; Augustson and Morken, 1996). Studies have shown that dentists experience more neck, shoulder and back pain compared to practitioners in other occupational groups (Kuorinka et al., 1987; van Doorn, 1995).

The work area of a dentist is narrow and because of this, dentists are subjected to very inflexible work posture during performance of dental treatment (Bramson, Smith and Romagnoli, 1998). Furthermore, precise motor skills are required during dental procedures to complete goaloriented tasks in a dynamic setting. The persistence and aggravation of pain could be related to many physical factors such as repetitive motion, awkward back postures, long period of static posture and precise hand and wrist movement (Graham, 2002; Alexopoulos, Stathi and Charizani, 2004; Lindfors, von Thiele and Lundberg, 2006). Psychological stress during procedures due to unexpected procedural challenges, time constraints and such and possible pre-existing pain conditions may also contribute to musculoskeletal pain among dental practitioners.

Previous studies suggest that musculoskeletal disorders among dental professionals start as early as during the course of clinical training period at dental school (Melis et al., 2004; Thornton et al., 2008). This is because dental students train in the same environment and adapt the same work behaviour as dental practitioners. However, students perform dental procedures without an assistant as four-handed dentistry is usually not available in a dental school clinical setting. Thus, students have to shift back and forth from the operating site to the tray table, reach instruments after instruments, twist, bend and contort their bodies in order to get closer to the treatment site. Today's generation of dental students have been reported with increased prevalence of back pain (Smith and Leggat, 2007). Melis et al reported that work-related musculoskeletal disorder among dental students occurs during the 4-year academic training period (Melis et al., 2004) . Reising et al concluded that $70 \%$ of dental students experienced pain in the third year (Rising et al., 2005). The development of chronic pain problems and the frequency and duration of which pain is maintained are greatly influenced by the methods of which students use to acquire dental skills since the first year of dental school.

Previously our department has published extensive research on various aspects of prosthetic dentistry
('Evaluation of Corrosive Behavior of Four Nickelchromium Alloys in Artificial Saliva by Cyclic Polarization Test:An in vitro Study', 2017; Ganapathy, Kannan and Venugopalan, 2017; Jain, 2017a, 2017b; Ranganathan, Ganapathy and Jain, 2017; Ariga et al., 2018; Gupta, Ariga and Deogade, 2018; Anbu et al., 2019; Ashok and Ganapathy, 2019; Duraisamy et al., 2019; Varghese, Ramesh and Veeraiyan, 2019), this vast research experience has inspired us to research the prevalence of musculoskeletal pain among dental students.

\section{MATERIAL AND METHODS}

This retrospective study involved the construction and distribution of questionnaires to dental students from a private dental hospital during a one-week period. The criterion for selection was third to fifth year undergraduate dental students. Students who have a history of trauma. rheumatoid arthritis, surgery of neck, shoulder, back and hands were excluded from the study. The survey was approved by the Ethical Committee Board of the dental hospital. Informed consent was obtained from all the participants and participation was voluntary without any incentives offered. The survey was done anonymously without identifiers of the participants.

The questionnaire included demographic information such as age and gender, and other variables such as year of study, presence of musculoskeletal pain, location of pain, characteristic of pain such as pain duration and intensity and frequency of weekly exercise. The location of pain was classified into 5 regions: neck/shoulder, upper back, lower back, thumb/hand and wrist. If more than one body region was identified as being painful, students marked the most symptomatic region. Pain characteristic was assessed by duration and intensity of pain. Pain duration was categorised to less than 1 hour, 1-3 hours and 4-8 hours per day. Pain intensity was divided into mild, moderate and severe. Frequency of weekly regular exercise was recorded as never, once a week, thrice a week and five times a week.

Statistical Analysis: All the data obtained were entered into Microsoft Office Excel (2013) and analysed using SPSS Software Version 26.0. Data were subjected to descriptive statistics in the form of frequency and percentages. Chi-square test was conducted to check for statistical significance between variables. Significance test level was set at $\mathrm{p}<0.05$.

\section{RESULTS AND DISCUSSION}

A total of 114 undergraduate dental students participated in the survey. This included $37(32.5 \%)$ males and 77 $(67.5 \%)$ females. Based on the year of study, 31 (27.2\%) were third years, 18 (15.8\%) were fourth years and 65 (57\%) were interns. Out of the total 114 participants, $71.1 \%$ reported with some type of musculoskeletal pain. Based on gender, musculoskeletal pain was observed in $80.5 \%$ of females and $51.4 \%$ of males and this result was statistically significant $(p<0.05)$ (Figure 1$)$. 
According to the year of study, the reported presence of pain was higher in interns (83.1\%), followed by fourth years (61.1\%) and third years (51.6\%). The percentage of participants reporting pain appeared to increase with the number of years in dental school and this was found to be statistically significant $(p<0.05)$ (Figure 2).

Figure 1: Bar chart denotes the association between presence of musculoskeletal pain and gender. $X$-axis represents the presence of musculoskeletal pain and $\mathrm{Y}$-axis represents the percentage of male and female students. Pearson Chi-Square value- 10.337 ; df- 1 ; $p$ value- 0.001 $(<0.05)$, hence, statistically significant. Presence of musculoskeletal pain was significantly higher in females (pink) compared to males.

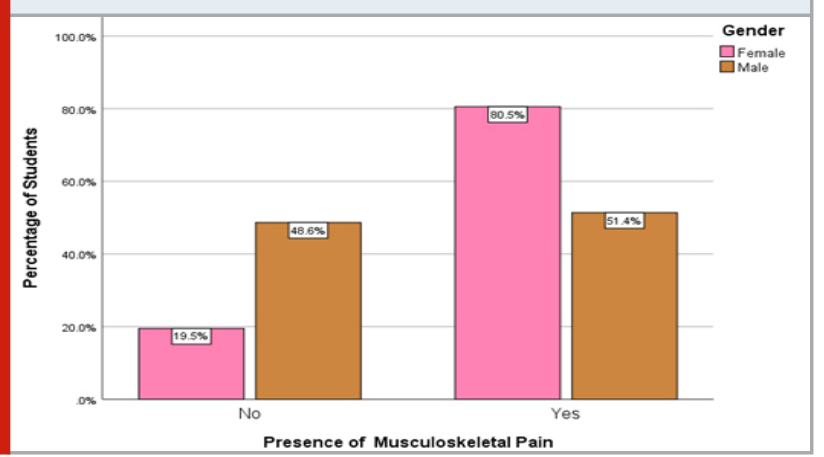

Figure 2: Bar chart denotes the association between presence of musculoskeletal pain and year of study. X-axis represents the presence of musculoskeletal pain and Y-axis represents the percentage of students based on year of study. Pearson Chi-Square value- 11.130 ; df- 2 ; p value$0.004(<0.05)$, hence, statistically significant. Presence of musculoskeletal pain was significantly higher in interns (grey) compared to final year and third year students.

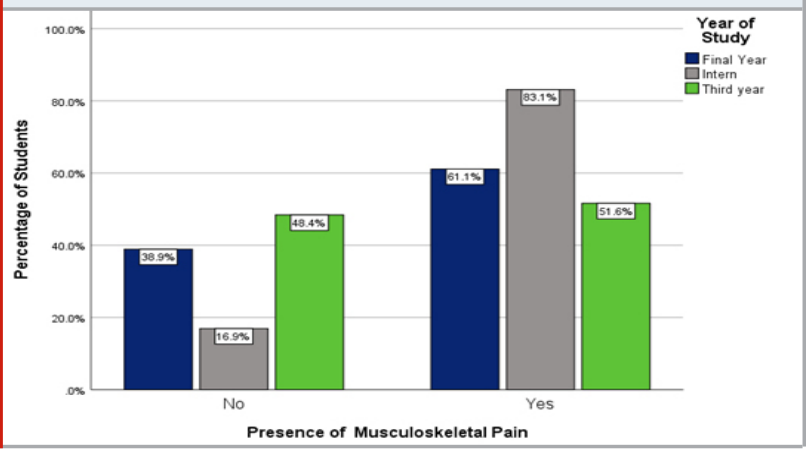

The order of most prevalent pain locations was the neck/shoulder (44.4\%), lower back (28.4\%), thumb/ hand (14.8\%), upper back (9.9\%) and wrist (2.5\%). However, the association between the location of pain and gender, and the location of pain and year of study was statistically not significant (p>0.05)(Figure 3)(Figure 4). Most participants $(59.3 \%)$ reported that the pain they experienced lasted for 1-3 hours and this was more common in females compared to males but this was statistically not significant ( $p>0.05$ )(Figure 5). Based on the year of study, the majority of interns $(64.8 \%)$ and final years $(63.6 \%)$ experienced musculoskeletal pain which lasted for 1-3 hours and most third years (62.5\%) had musculoskeletal pain which lasted for less than an hour. The association between duration of pain and year of study was statistically significant $(p<0.05)$ (Figure 6). 65.4\% of participants with musculoskeletal pain reported the intensity of pain was mild, whereas $33.3 \%$ experienced moderate pain and $1.2 \%$ experienced severe pain.

Figure 3: Bar chart denotes the association between location of musculoskeletal pain and gender. $\mathrm{X}$-axis represents the location of musculoskeletal pain and Y-axis represents the percentage of male and female students. Pearson Chi-Square value- 1.885 ; df- 4 ; p value- 0.757 (>0.05), hence, statistically not significant. Males (brown) experience more pain in the neck/shoulder and thumb/ hand region compared to females but the result was statistically not significant.

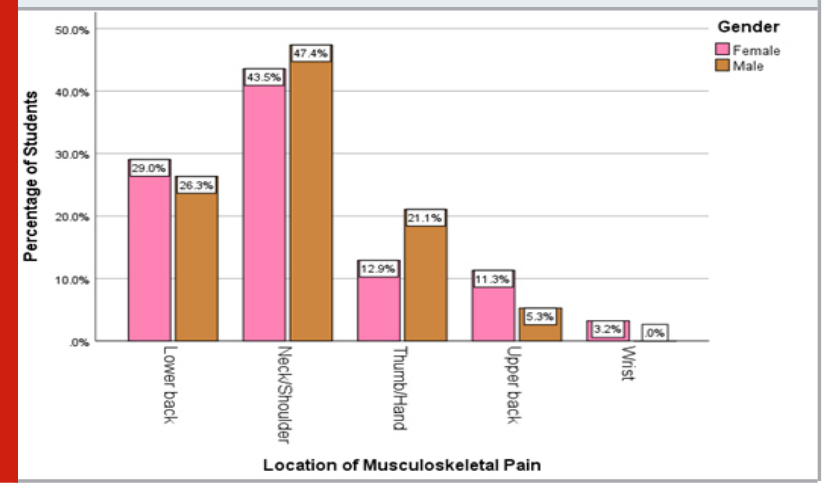

Figure 4. Bar chart denotes the association between location of musculoskeletal pain and year of study. X-axis represents the location of musculoskeletal pain and Y-axis represents the percentage of students based on year of study. Pearson Chi-Square value- 14.469 ; df- 8; $p$ value0.070 (>0.05), hence, statistically not significant. Pain in the neck/shoulder and wrist region was more among interns (grey) whereas lower back pain was higher among final year (blue) students but the result was statistically not significant.

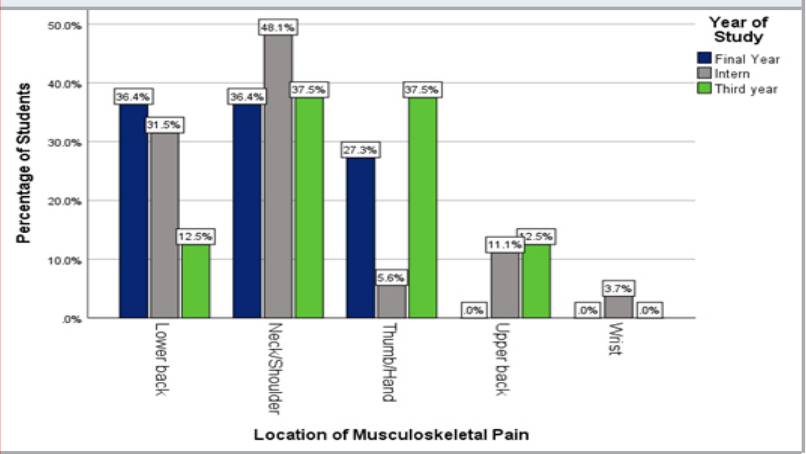

The association between intensity of pain and gender, and between intensity of pain and year of study was statistically not significant $(\mathrm{p}>0.05)$ (Figure 7)(Figure 8). Presence of musculoskeletal pain was significantly higher among those who do not engage in exercise or any 
physical activity (80.9\%) compared to those who exercise once and three times a week $(\mathrm{p}<0.05)($ Figure 9$)$. This study evaluated the prevalence of musculoskeletal pain in a sample of dental students at a private dental hospital. Many research studies have reported high prevalence of work-related musculoskeletal disorder among dentists. Dental students were no exception. The results of this study confirmed the presence of musculoskeletal pain among dental students.

Figure 5: Bar chart denotes the association between duration of musculoskeletal pain and gender. $\mathrm{X}$-axis represents the duration of musculoskeletal pain and $\mathrm{Y}$-axis represents the percentage of male and female students. Pearson Chi-Square value- 1.640; df- 2; $p$ value- 0.440 ( $>0.05)$, hence, statistically not significant. Most musculoskeletal pain lasted for 1-3 hours and this was more common in females (pink) compared to males but the result was statistically not significant.

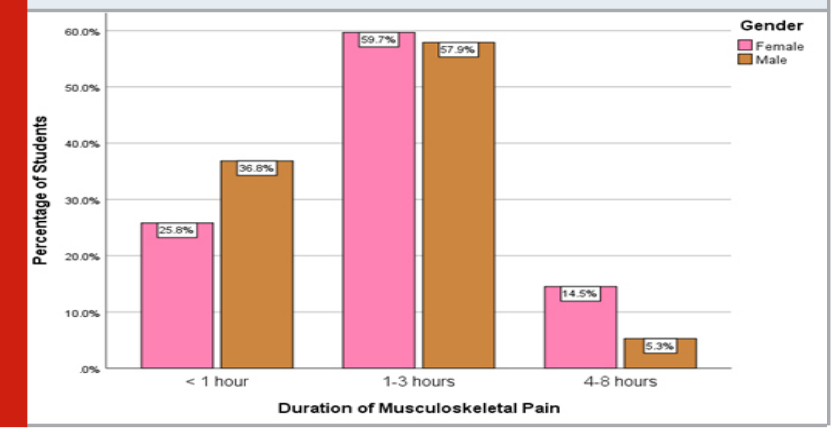

Figure 6: Bar chart denotes the association between duration of musculoskeletal pain and year of study. $\mathrm{X}$-axis represents the duration of musculoskeletal pain and Y-axis represents the percentage of students based on year of study. Pearson Chi-Square value- 16.011; df4 ; p value- $0.003(<0.05)$, hence, statistically significant. Musculoskeletal pain which lasted for 1-3 hours were significantly higher among interns compared to final year and third year students.

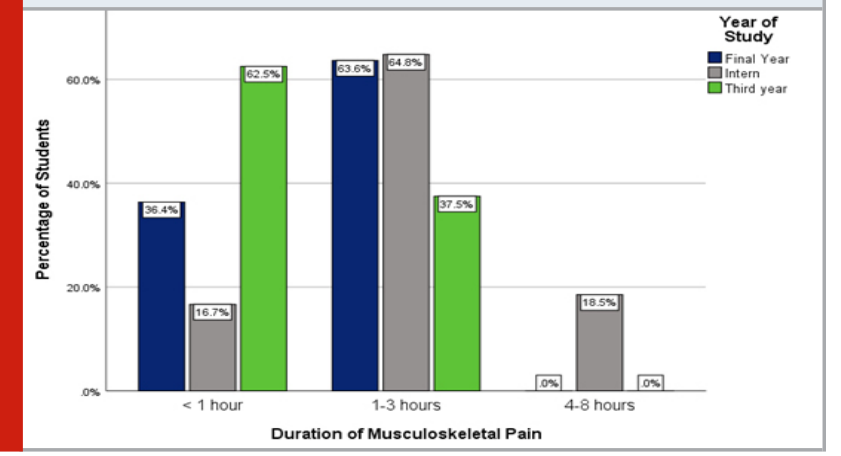

The survey revealed that $71.1 \%$ of students reported some type of musculoskeletal pain. This confirmed an early study by Rising et al on their reports of body pain in a dental student population which concluded $46-71 \%$ of dental students reported body pain (Rising et al., 2005). Movahhed et al studied the musculoskeletal pain report among Mashhad dental students and found that most 366 undergraduate dental students (81.6\%) reported pain at least in one region of the body (Movahhed et al., 2013). A study on the perceived musculoskeletal symptoms among dental students in the clinic work environment by Thornton et al also found that the majority of dental students had musculoskeletal pain (Thornton et al., 2008). The studies were in agreement with our current findings.

Figure 7: Bar chart denotes the association between intensity of musculoskeletal pain and gender. $\mathrm{X}$-axis represents the intensity of musculoskeletal pain and $\mathrm{Y}$-axis represents the percentage of male and female students. Intensity of musculoskeletal pain was mild for most male and female students. Pearson Chi-Square value- 0.363; df- 2 ; p value- $0.834(>0.05)$, hence, statistically not significant. More males (brown) experienced mild intensity of musculoskeletal pain compared to females but this was statistically not significant.

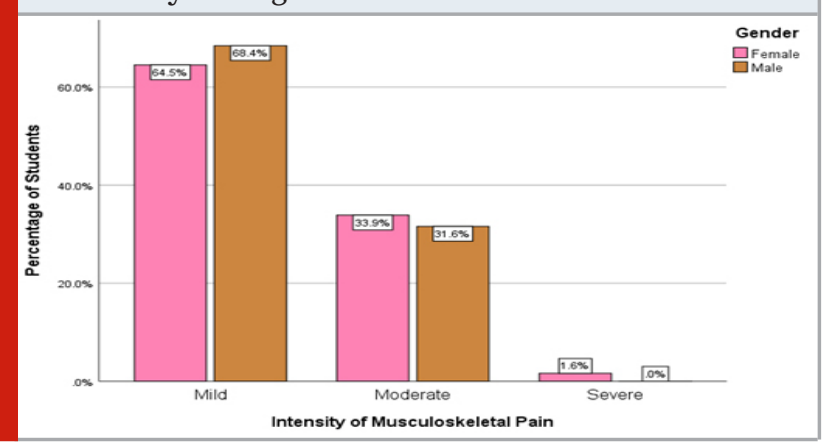

Figure 8: Bar chart denotes the association between intensity of musculoskeletal pain and year of study. $\mathrm{X}$-axis represents the intensity of musculoskeletal pain and Y-axis represents the percentage of students based on year of study. Pearson Chi-Square value- 9.211 ; df- 4 ; $p$ value- 0.056 (>0.05), hence, statistically not significant. More third years (green) experienced mild intensity of musculoskeletal pain compared to interns and final years but this was statistically not significant.

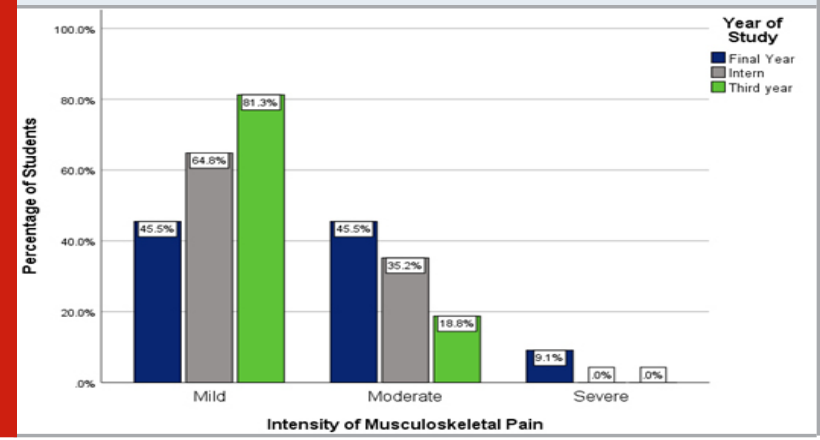

The current study found that musculoskeletal pain was higher in female dental students compared to males students. In 2006, Lindfors et al reported that female dentists showed a higher prevalence of musculoskeletal pain (Lindfors, von Thiele and Lundberg, 2006), which coincided with our findings. Movahhed et al also reported the majority of dental students with body 
pain were females (Movahhed et al., 2013). A past study on the prevalence and risk factor associated with musculoskeletal pain among students of MGM Dental College found that more male dental students experienced musculoskeletal pain compared to female students (Madaan and Chaudhari, 2012) and this did not coincide with the current study.

Figure 9: Bar chart denotes the association between presence of musculoskeletal pain and frequency of weekly exercise. $\mathrm{X}$-axis represents the presence of musculoskeletal pain and Y-axis represents the percentage of student's frequency of weekly exercise by students. Pearson ChiSquare value- 13.390; df- 3; p value- $0.006(<0.05)$, hence, statistically significant. Significantly more students who did not experience musculoskeletal pain exercise five times a week whereas most students who experience musculoskeletal pain never engage in weekly exercise.

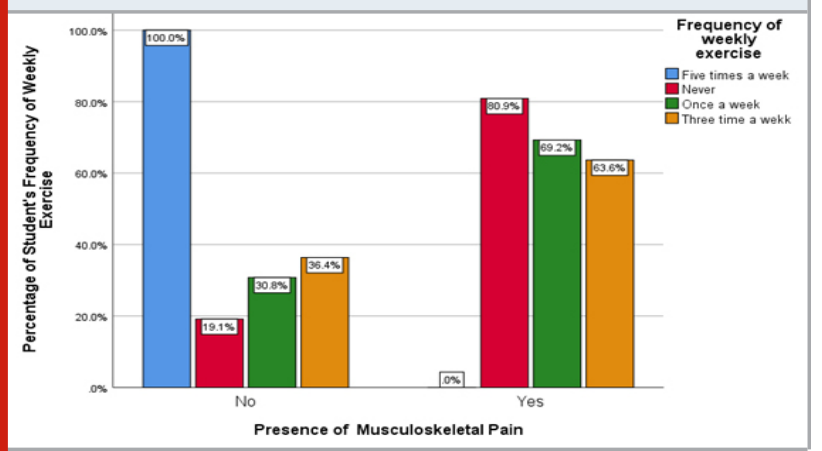

According to our results, a higher percentage of interns experienced musculoskeletal pain, followed by fourth years and third years. Musculoskeletal pain appeared to increase with the number of years in dental school. This finding coincided with a study by Rising et al who also found body pain to be more prevalent in interns and least prevalent in third year students (Rising et al., 2005). Interns (84\%) were also reported to experience more body pain compared to fourth year (81\%) and third year (78\%) students in a study by Madaan et al (Madaan and Chaudhari, 2012).

We found that the most common area affected by pain was the neck/shoulder region (44.4\%), followed by the lower back region (28.4\%). Rising et al also reported a similar finding which stated the most prevalent location of pain reported by dental students were neck/shoulder region and back pain (Rising et al., 2005). Prevalence of neck/shoulder pain in our survey was almost similar to the results of a study by Morse et al in 2007 in which $37 \%$ of students reported neck pain (Morse et al., 2007). Melis et al found the prevalence of neck pain among Italian dental students as $40.4 \%$ which was similar to our study (Melis et al., 2004).

The results of this study showed that dental students are at a risk for development of musculoskeletal disorders. Strategies for reducing musculoskeletal disorder should be elucidated before it affects a large number of dentists. It is of utmost importance to educate students on proper ergonomics during clinical procedures as well as musculoskeletal cares.

\section{CONCLUSION}

Within the limits of this study, it was observed that there was a high prevalence of musculoskeletal pain among dental students and prevalence was higher among females and dental interns. The most common area affected by pain was the neck/shoulder region.

\section{ACKNOWLEDGEMENTS}

We would like to express sincere gratitude to Saveetha Dental College and Hospital, Chennai for the contribution and involvement in this study.

Conflict of Interest: No conflict of interest has been declared by the authors.

\section{REFERENCES}

Alexopoulos, E. C., Stathi, I.-C. and Charizani, F. (2004) 'Prevalence of musculoskeletal disorders in dentists', BMC musculoskeletal disorders, 5, p. 16.

Anbu, R. T. et al. (2019) 'Comparison of the Efficacy of Three Different Bone Regeneration Materials: An Animal Study', European journal of dentistry, 13(1), pp. 22-28.

Ariga, P. et al. (2018) 'Determination of Correlation of Width of Maxillary Anterior Teeth using Extraoral and Intraoral Factors in Indian Population: A Systematic Review', World Journal of Dentistry, 9(1), pp. 68-75.

Ashok, V. and Ganapathy, D. (2019) 'A geometrical method to classify face forms', Journal of oral biology and craniofacial research, 9(3), pp. 232-235.

Augustson, T. E. and Morken, T. (1996) 'Musculoskeletal problems among dental health personnel. A survey of the public dental health services in Hordaland, Tidsskrift for den Norske laegeforening: tidsskrift for praktisk medicin, ny raekke, 116(23), pp. 2776-2780.

Bramson, J. B., Smith, S. and Romagnoli, G. (1998) 'Evaluating dental office ergonomic. Risk factors and hazards', Journal of the American Dental Association , 129(2), pp. 174-183.

van Doorn, J. W. C. (1995) 'Low back disability among self-employed dentists, veterinarians, physicians and physical therapists in The Netherlands: a retrospective study over a 13 -year period $(\mathrm{N}=1,119)$ and an early intervention program with 1-year follow-up ( $\mathrm{N}=134)$ ', Acta orthopaedica Scandinavica. Taylor \& Francis, 66(sup263), pp. 3-64.

Duraisamy, R. et al. (2019) 'Compatibility of Nonoriginal Abutments With Implants: Evaluation of Microgap at the Implant-Abutment Interface, With Original and Nonoriginal Abutments', Implant dentistry, 28(3), pp. 289-295.

Evaluation of Corrosive Behavior of Four Nickelchromium Alloys in Artificial Saliva by Cyclic Polarization Test:An in vitro Study' (2017) World 
Journal of Dentistry, 8(6), pp. 477-482.

Ganapathy, D. M., Kannan, A. and Venugopalan, S. (2017) 'Effect of Coated Surfaces influencing Screw Loosening in Implants: A Systematic Review and Meta-analysis', World Journal of Dentistry, 8(6), pp. 496-502.

Graham, C. (2002) 'Ergonomics in dentistry, Part 1', Dentistry today, 21(4), pp. 98-103.

Gupta, P., Ariga, P. and Deogade, S. C. (2018) 'Effect of Monopoly-coating Agent on the Surface Roughness of a Tissue Conditioner Subjected to Cleansing and Disinfection: A Contact Profilometric Study', Contemporary clinical dentistry, 9(Suppl 1), pp. S122S126.

Hayes, M., Cockrell, D. and Smith, D. R. (2009) 'A systematic review of musculoskeletal disorders among dental professionals', International journal of dental hygiene, 7(3), pp. 159-165.

Jain, A. R. (2017a) 'Clinical and Functional Outcomes of Implant Prostheses in Fibula Free Flaps', World Journal of Dentistry, 8(3), pp. 171-176.

Jain, A. R. (2017b) 'Prevalence of Partial Edentulousness and Treatment needs in Rural Population of South India', World Journal of Dentistry, 8(3), pp. 213-217.

Kuorinka, I. et al. (1987) 'Standardised Nordic questionnaires for the analysis of musculoskeletal symptoms', Applied ergonomics, 18(3), pp. 233-237.

Leggat, P. A., Kedjarune, U. and Smith, D. R. (2007) 'Occupational health problems in modern dentistry: a review', Industrial health, 45(5), pp. 611-621.

Lindfors, P., von Thiele, U. and Lundberg, U. (2006) 'Work characteristics and upper extremity disorders in female dental health workers', Journal of occupational health, 48(3), pp. 192-197.

Madaan, V. and Chaudhari, A. (2012) 'Prevalence and Risk Factor associated with Musculoskeletal Pain among Students of MGM Dental College: A Cross- Sectional Survey', Journal of Contemporary Dentistry, pp. 22-27. doi: 10.5005/jp-journals-10031-1004.

Melis, M. et al. (2004) 'Upper body musculoskeletal symptoms in Sardinian dental students', Journal , 70(5), pp. 306-310.

Morse, T. et al. (2007) 'Musculoskeletal disorders of the neck and shoulder in dental hygienists and dental hygiene students', Journal of dental hygiene: JDH / American Dental Hygienists' Association, 81(1), p. 10. Movahhed, T. et al. (2013) 'Musculoskeletal pain reports among Mashhad dental students, Iran', Pakistan journal of biological sciences: PJBS, 16(2), pp. 80-85.

Murtomaa, H. (1982) 'Work-related complaints of dentists and dental assistants', International archives of occupational and environmental health, 50(3), pp. 231-236.

Pollack, R. (1996) 'Dental office ergonomics: how to reduce stress factors and increase efficiency', Journal, 62(6), pp. 508-510.

Ranganathan, H., Ganapathy, D. M. and Jain, A. R. (2017) 'Cervical and Incisal Marginal Discrepancy in Ceramic Laminate Veneering Materials: A SEM Analysis', Contemporary clinical dentistry, 8(2), pp. 272-278.

Rising, D. W. et al. (2005) 'Reports of body pain in a dental student population', Journal of the American Dental Association , 136(1), pp. 81-86.

Smith, D. R. and Leggat, P. A. (2007) 'Back Pain in the Young: A Review of Studies Conducted Among School Children and University Students', Current pediatric reviews, 3(1), pp. 69-77.

Stockstill, J. W. et al. (1993) 'Prevalence of upper extremity neuropathy in a clinical dentist population', Journal of the American Dental Association , 124(8), pp. 67-72.

Thornton, L. J. et al. (2008) 'Perceived musculoskeletal symptoms among dental students in the clinic work environment', Ergonomics, 51(4), pp. 573-586.

Varghese, S. S., Ramesh, A. and Veeraiyan, D. N. (2019) 'Blended Module-Based Teaching in Biostatistics and Research Methodology: A Retrospective Study with Postgraduate Dental Students', Journal of dental education, 83(4), pp. 445-450. 